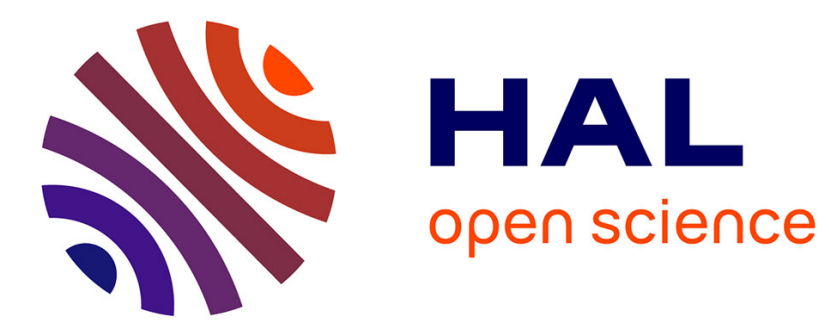

\title{
A preventive maintenance policy for a continuously monitored system with correlated wear indicators
}

Sophie Mercier, Ha Pham

\section{To cite this version:}

Sophie Mercier, Ha Pham. A preventive maintenance policy for a continuously monitored system with correlated wear indicators. European Journal of Operational Research, 2012, 222 (2), pp.263272. 10.1016/j.ejor.2012.05.011 . hal-00864795

\section{HAL Id: hal-00864795 \\ https://hal.science/hal-00864795}

Submitted on 14 Mar 2018

HAL is a multi-disciplinary open access archive for the deposit and dissemination of scientific research documents, whether they are published or not. The documents may come from teaching and research institutions in France or abroad, or from public or private research centers.
L'archive ouverte pluridisciplinaire HAL, est destinée au dépôt et à la diffusion de documents scientifiques de niveau recherche, publiés ou non, émanant des établissements d'enseignement et de recherche français ou étrangers, des laboratoires publics ou privés. 


\title{
A preventive maintenance policy for a continuously monitored system with correlated wear indicators
}

\author{
Sophie MERCIER, Hai Ha PHAM \\ Université de Pau et des Pays de l'Adour,
}

Laboratoire de Mathématiques et de leurs Applications - Pau (UMR CNRS 5142),

Bâtiment IPRA, Avenue de l'Université, F-64013 PAU cedex, FRANCE

sophie.mercier@univ-pau.fr, haiha.pham@etud.univ-pau.fr

March 9, 2012

\begin{abstract}
A continuously monitored system is considered, that gradually and stochastically deteriorates according to a bivariate non decreasing Lévy process. The system is considered as failed as soon as its bivariate deterioration level enters a failure zone, assumed to be an upper set. A preventive maintenance policy is proposed, which involves a delayed replacement, triggered by the reaching of some preventive zone for the system deterioration level. The preventive maintenance policy is assessed through a cost function on an infinite horizon time.
\end{abstract}

${ }^{*}$ Corresponding author, Phone: 335594075 37, Fax: 33559407555 
The cost function is provided in full form, and tools are provided for its numerical computation. The influence of different parameters on the cost function is studied, both from a theoretical and/or numerical point of view.

Keywords: Reliability; multivariate Lévy processes; dependent wear indicators; Gamma processes; optimal replacement; renewal theory.

\section{Introduction}

One major concern in reliability is the study of preventive maintenance policies, which aims at enlarging systems lifetimes and/or reducing their operating costs. With that purpose, a first point is to propose a stochastic model for the system deterioration. In case of a random non decreasing deterioration, classical models include compound Poisson processes and Gamma processes, according to whether the deterioration is due to isolated shocks or to some continuous wear accumulation, see Abdel-Hameed (1975), Van Noortwijk (2009) or Singpurwalla (1995) e.g.. Such classical models are univariate non decreasing Lévy processes. One single indicator may however be insufficient to measure a system deterioration, see Mercier \& al. (2011) for an industrial example, where two indicators are necessary to describe the deterioration level of a railway track geometry. In this example, the traffic on the track influences the evolution of both indicators, which implies some correlation between them. More generally, indicators measuring different aspects of a same system are likely to present some dependency. Hence the need for multivariate wear indicators. Such models also are of interest in the case of univariate indicators measuring the deterioration levels of different systems submitted to some common stressing environment.

Up to our knowledge, multivariate non decreasing wear indicators have 
not been much studied in the previous literature. Two notable exceptions may however be found in Buijs \& al. (2005) and Ebrahimi (2004), which both use specific constructions leading to some specific bivariate increasing Lévy processes (though not recognized as such in the quoted papers). We here propose to model the system deterioration level by a general increasing bivariate Lévy process (or bivariate subordinator).

Under such an assumption, a system is considered, subject to continuous monitoring. It is considered as failed as soon as its bivariate deterioration level has reached a failure zone $\mathcal{L}$. Once in $\mathcal{L}$, the system cannot leave $\mathcal{L}$ without being repaired. This property is translated through the assumption that $\mathcal{L}$ is an upper set. As in Bérenguer \& al. (2003), when the system enters $\mathcal{L}$, a signal is immediately sent to a repair team. It takes some delay $\tau$ for the repair team to arrive. The repair duration is short compared to the delay $\tau$ and is hence considered as instantaneous (and perfect). To shorten the system down-time, a preventive maintenance (PM) policy is proposed, where the signal is sent to the repair team as soon as the deterioration level reaches a PM zone $\mathcal{M}$, larger than $\mathcal{L}$.

The point of the paper is the study of the PM policy, which is classically assessed through a cost function on an infinite time horizon. Another point of interest is the influence of different parameters on the PM efficiency. The influence of the shape of the upper sets $\mathcal{L}$ and $\mathcal{M}$ is studied too.

The paper is organized as follows: in Section 2, the model is presented, both for the initial (without maintenance) and preventively maintained system. Section 3 is devoted to theoretical developments whereas Section 4 presents some numerical experiments. We finally conclude in Section 5. 


\section{The model}

\subsection{The initial system}

A system is considered, with deterioration level measured by a bivariate non decreasing process $\left(X_{t}=\left(X_{t}^{(1)}, X_{t}^{(2)}\right)\right)_{t \geq 0}$. The process $\left(X_{t}\right)_{t \geq 0}$ is assumed to be a non decreasing Lévy process (or bivariate subordinator), namely a process with range $\mathbb{R}_{+}^{2}$, starting from $(0,0)$, which is right continuous with left-side limits and stochastically continuous, and has stationary and independent increments. The Brownian part of a subordinator is null and its drift belongs to $\mathbb{R}_{+}^{2}$. The drift part does not bring much to the present study and it is consequently assumed in the sequel that $\left(X_{t}\right)_{t \geq 0}$ is a bivariate subordinator with null drift, namely a pure jump process, see Bertoin (1996) or Sato (1999) e.g.. To avoid trivialities, the process $\left(X_{t}\right)_{t \geq 0}$ is also assumed to be non zero: $\mathbb{P}\left(X_{t}^{(1)}>0, X_{t}^{(2)}>0\right)>0$. Such assumptions will be referred to as assumption $\mathcal{H}$. For $i=1,2$, the marginal process $\left(X_{t}^{(i)}\right)_{t \geq 0}$ is known to be an univariate subordinator (with null drift).

The system is continuously and perfectly monitored. It is considered as failed as soon as its bivariate deterioration level reaches a failure zone $\mathcal{L} \subset \mathbb{R}_{+}^{2}$. The failure time of the unmaintained system hence is:

$$
\sigma_{\mathcal{L}}=\inf \left\{t \geq 0 \mid X_{t} \in \mathcal{L}\right\}
$$

As explained in the introduction, $\mathcal{L}$ is assumed to be a closed and non empty upper set, namely such that for all $\left(x_{1}, x_{2}\right) \in \mathcal{L}$ and all $\left(y_{1}, y_{2}\right) \in \mathbb{R}_{+}^{2}$, if $\left(y_{1}, y_{2}\right) \geq\left(x_{1}, x_{2}\right)$, then $\left(y_{1}, y_{2}\right) \in \mathcal{L}$. As $\left(X_{t}\right)_{t \geq 0}$ is non decreasing, this means that once failed, the system cannot leave $\mathcal{L}$ any more and remains failed (until it is repaired).

For illustrative purpose, three different shapes are envisioned for $\mathcal{L}$. For the first two shapes, the system may be considered as composed of two dif- 
ferent units and for $i=1,2$, the marginal process $\left(X_{t}^{(i)}\right)_{t \geq 0}$ stands for the deterioration level of the $i$-th unit. Setting $L_{i}>0$ to be the failure threshold for the $i$-th unit, the corresponding univariate failure time is

$$
\sigma_{L_{i}}^{(i)}=\inf \left\{t \geq 0 \mid X_{t}^{(i)} \geq L_{i}\right\}
$$

Two classical structures are then envisioned for the two-units system, which leads to the following first two cases:

Case 1 The two units are set up into series. The time-to-failure of the whole system then is:

$$
\begin{aligned}
\min \left(\sigma_{L_{1}}^{(1)}, \sigma_{L_{2}}^{(2)}\right) & =\inf \left\{t \geq 0 \mid X_{t}^{(1)} \geq L_{1} \text { or } X_{t}^{(2)} \geq L_{2}\right\} \\
& =\inf \left\{t \geq 0 \mid X_{t} \notin\left[0, L_{1}\left[\times\left[0, L_{2}[\}\right.\right.\right.\right. \\
& =\sigma_{\mathcal{L}},
\end{aligned}
$$

with $\mathcal{L}=\mathbb{R}_{+}^{2} \backslash\left[0, L_{1}\left[\times\left[0, L_{2}[\right.\right.\right.$.

Case 2 The two units are set up into parallel. The time-to-failure of the whole system is $\max \left(\sigma_{L_{1}}^{(1)}, \sigma_{L_{2}}^{(2)}\right)=\sigma_{\mathcal{L}}$, with $\mathcal{L}=\left[L_{1}, \infty\left[\times\left[L_{2}, \infty[\right.\right.\right.$.

Case 3 Both components of $\left(X_{t}\right)_{t \geq 0}$ stand for different indicators of a single system and the system time to failure is

$$
\inf \left\{t \geq 0 \mid X_{t}^{(1)}+X_{t}^{(2)} \geq L\right\}=\sigma_{\mathcal{L}}
$$

with $\mathcal{L}=\left\{\left(x_{1}, x_{2}\right) \in \mathbb{R}_{+}^{2} \mid x_{1}+x_{2} \geq L\right\}$

Such three shapes are plotted in Figure 1.

Once the system is failed, a signal is sent to the repair team and an instantaneous repair takes place at time $\sigma_{\mathcal{L}}+\tau$, where $\tau$ is the deterministic time required by the repair team to arrive (the delay). The repair is perfect, which means that at repair, both of the system deterioration indicators are reset to zero. 


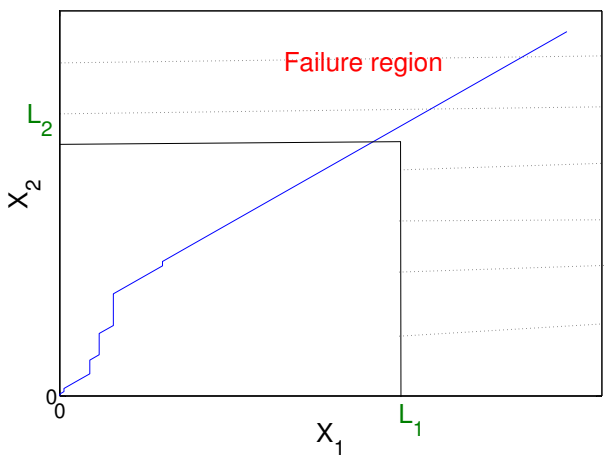

(a) The first shape

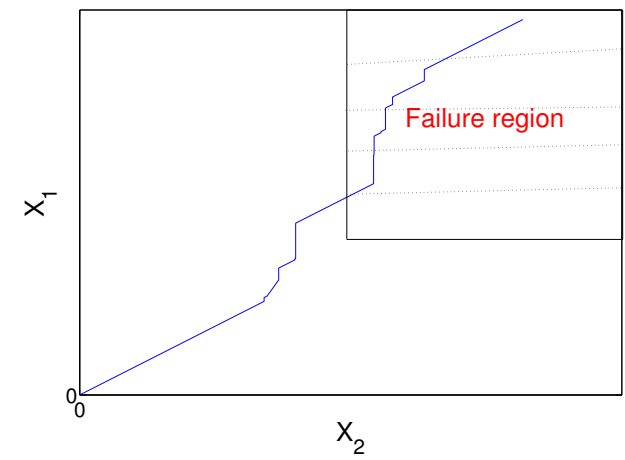

(b) The second shape

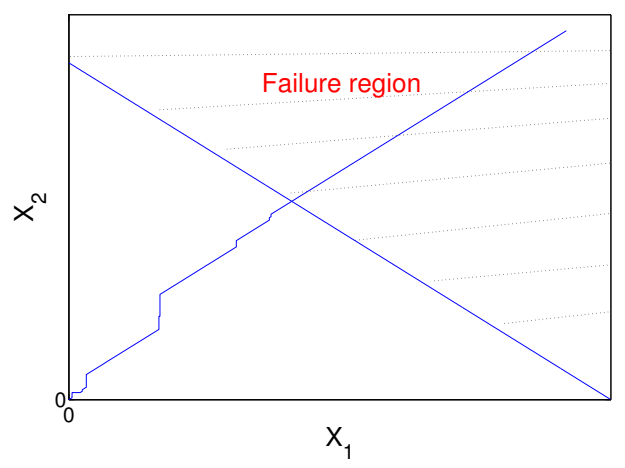

(c) The third shape

Figure 1: Examples of failure regions

\subsection{The preventive maintenance policy}

Without any PM policy, the system is down from $\sigma_{\mathcal{L}}$ up to $\sigma_{\mathcal{L}}+\tau$. To shorten this down-time (of length $\tau$ ), the following PM policy is applied: setting $\mathcal{M}$ to be a closed and non empty upper set such that $\mathcal{L} \subset \mathcal{M} \subset \mathbb{R}_{+}^{2}$, a signal is preventively sent to the repair team at time $\sigma_{\mathcal{M}}\left(\leq \sigma_{\mathcal{L}}\right)$. The system is then perfectly and instantaneously repaired at time $\sigma_{\mathcal{M}}+\tau$. If $\sigma_{\mathcal{L}}<\sigma_{\mathcal{M}}+\tau$, a failure occurs before the repair and the down-time duration is $\sigma_{\mathcal{M}}+\tau-\sigma_{\mathcal{L}}$. On the contrary, if $\sigma_{\mathcal{L}} \geq \sigma_{\mathcal{M}}+\tau$, the system is repaired before failure and there is no down-time up to the repair. In each case, the down time up to 
the repair hence is $\left(\sigma_{\mathcal{M}}+\tau-\sigma_{\mathcal{L}}\right)^{+}=\max \left(\sigma_{\mathcal{M}}+\tau-\sigma_{\mathcal{L}}, 0\right)$.

The future evolution of the system after repair is assumed to be independent from its past, and stochastically identical to its initial evolution. Setting $\left(Z_{t}\right)_{t \geq 0}$ to be the process describing the maintained system, $\left(Z_{t}\right)_{t \geq 0}$ appears as a regenerative process with cycles delimited by repairs (and $t=0$ ) and generic cycle length $\sigma_{\mathcal{M}}+\tau$. This is illustrated in Figure 2 where the horizontal axis corresponds to the time and the vertical one to the deterioration level, drawn as a one-dimensional level for sake of clarity. Note that, as a bivariate subordinator (with null drift) is a pure jump process, the failure zone $\mathcal{L}$ has a non zero probability to be reached at the same time as the system enters $\mathcal{M}$. This can be seen in Figure 2, where $\sigma_{\mathcal{M}}=\sigma_{\mathcal{L}}$ in the first cycle $\left(\sigma_{\mathcal{M}}^{(1)}=\sigma_{\mathcal{L}}^{(1)}\right)$. In the second cycle (which starts at $\left.\sigma_{\mathcal{M}}^{(1)}+\tau\right)$, the system is replaced before failure $\left(\sigma_{\mathcal{M}}^{(2)}+\tau<\sigma_{\mathcal{L}}^{(2)}\right)$.

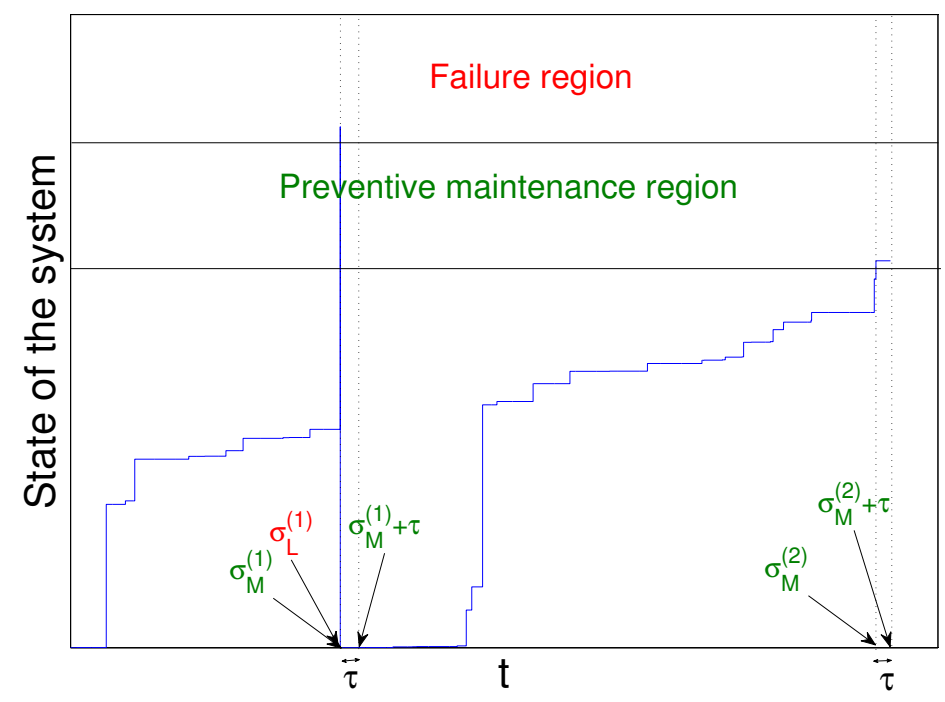

Figure 2: The preventive maintenance policy

Taking $\mathcal{M}=\mathcal{L}$, the unmaintained system appears as a special case of the maintained system. Taking $\mathcal{M}=\mathbb{R}_{+}^{2}$ provides $\sigma_{\mathcal{M}}=0$ and the system is 
replaced every $\tau$ time units. The classical periodic replacement policy with no repair at failure and period $\tau$ (Barlow \& Proschan (1965)) then appears as a special case of the PM policy.

To assess the PM policy, a cost function is considered, which takes into account:

- $C_{1}>0$ : the restoration cost of the system,

- $C_{2}>0$ : the unitary cost (per unit time) for down-time.

The envisioned cost function is the asymptotic unitary cost (per unit time), namely the function $C_{\infty}$ defined by:

$$
C_{\infty}=\lim _{t \rightarrow \infty} \frac{C(t)}{t} \text { a.s. }
$$

where $C(t)$ stands for the accumulated cost on the time interval $[0, t]$. Our goal is to prove existence of $C_{\infty}$, find a computable expression for it and study its behavior with respect to different parameters.

We will sometimes complete the assessment of the PM policy by another criterion, the asymptotic availability, defined by:

$$
A_{\infty}=\lim _{t \rightarrow \infty} \frac{U(t)}{t} \text { a.s. }
$$

where $U(t)$ stands for the system up-time on $[0, t]$. Methods are quite similar for both criteria and details are only provided for $C_{\infty}$.

\section{Theoretical results}

\subsection{Calculation of the cost function}

In order to prove the existence of the asymptotic unitary cost $C_{\infty}$, we use time-average properties from Asmussen (2003), which require $\mathbb{E}\left(\sigma_{\mathcal{M}}\right)$ to be finite. We first check this property. 
Lemma 1 Under assumption $\mathcal{H}$, the means of $\sigma_{\mathcal{L}}$ and $\sigma_{\mathcal{M}}$ are finite.

Proof. Let $A=\left(x_{1}, x_{2}\right) \in \mathcal{L}$ be fixed and let

$$
T_{A}=\inf \left\{t \geq 0 \mid X_{t}^{(1)} \geq x_{1}, X_{t}^{(2)} \geq x_{2}\right\}=\sup \left(\sigma_{x_{1}}^{(1)}, \sigma_{x_{2}}^{(2)}\right) .
$$

We have $\sigma_{\mathcal{M}} \leq \sigma_{\mathcal{L}} \leq T_{A} \leq \sigma_{x_{1}}^{(1)}+\sigma_{x_{2}}^{(2)}$ and it is sufficient to prove that $\mathbb{E}\left(\sigma_{x_{i}}^{(i)}\right)<+\infty$ for $i=1,2$. As we have not been able to find a reference for it, we prove this last condition here: we first note that

$$
\begin{aligned}
\mathbb{E}\left(\sigma_{x_{i}}^{(i)}\right) & =\int_{0}^{+\infty} \mathbb{P}\left(\sigma_{x_{i}}^{(i)}>t\right) d t=\int_{0}^{+\infty} \mathbb{P}\left(X_{t}^{(i)}<x_{i}\right) d t \\
& =\int_{0}^{+\infty} \mathbb{P}\left(e^{-X_{t}^{(i)}}>e^{-x_{i}}\right) d t \\
& \leq e^{x_{i}} \int_{0}^{+\infty} \mathbb{E}\left(e^{-X_{t}^{(i)}}\right) d t
\end{aligned}
$$

using Markov inequality for the last line. We next recall that the Laplace transform of the (univariate) subordinator $\left(X_{t}^{(i)}\right)_{t \geq 0}$ is provided by

$$
\mathbb{E}\left(e^{-X_{t}^{(i)}}\right)=e^{-t \phi^{(i)}(1)}
$$

where $\phi^{(i)}(1)=-\ln \left(\mathbb{E}\left(e^{-X_{1}^{(i)}}\right)\right)$. As $\mathbb{P}\left(X_{1}^{(i)}>0\right) \geq \mathbb{P}\left(X_{1}^{(i)}>0, X_{1}^{(2)}>0\right)>$ 0 by assumption, we first derive that $\phi^{(i)}(1)>0$ and next that $\mathbb{E}\left(\sigma_{x_{i}}^{(i)}\right)<$ $+\infty$ due to (1), which achieves the proof.

Proposition 2 Under assumption $\mathcal{H}$, the asymptotic unitary cost exists a.s. and is equal to

$$
C_{\infty}=\frac{C_{1}+C_{2} \mathbb{E}\left[\left(\sigma_{\mathcal{M}}+\tau-\sigma_{\mathcal{L}}\right)^{+}\right]}{\tau+\mathbb{E}\left(\sigma_{\mathcal{M}}\right)}
$$

Proof. The process $\left(Z_{t}\right)_{t \geq 0}$ is a regenerative process with generic cycle length $\sigma_{\mathcal{M}}+\tau$ and finite expected length, due to Lemma 1. We hence derive from Asmussen (2003) the existence of $C_{\infty}$, with

$$
C_{\infty}=\frac{\mathbb{E}\left[C\left(\sigma_{\mathcal{M}}+\tau\right)\right]}{\mathbb{E}\left(\sigma_{\mathcal{M}}+\tau\right)} .
$$


Besides, in a generic cycle, we have to pay $C_{1}$ for the restoration of the system and $C_{2}$ per unit down-time. This provides the result, recalling that the down-time duration is $\left(\sigma_{\mathcal{M}}+\tau-\sigma_{\mathcal{L}}\right)^{+}$, see Subsection 2.2.

Noting that

$\mathbb{E}\left[\left(\sigma_{\mathcal{M}}+\tau-\sigma_{\mathcal{L}}\right)^{+}\right]=\mathbb{E}\left(\int_{0}^{+\infty} \mathbf{1}_{\left\{\left(\sigma_{\mathcal{M}}+\tau-\sigma_{\mathcal{L}}\right)^{+}>u\right\}} d u\right)=\mathbb{E}\left(\int_{0}^{\tau} \mathbf{1}_{\left\{\sigma_{\mathcal{M}}+\tau-\sigma_{\mathcal{L}}>u\right\}} d u\right)$

and setting $t=\tau-u$, one gets:

$$
\mathbb{E}\left[\left(\sigma_{\mathcal{M}}+\tau-\sigma_{\mathcal{L}}\right)^{+}\right]=\mathbb{E}\left(\int_{0}^{\tau} \mathbf{1}_{\left\{t>\sigma_{\mathcal{L}}-\sigma_{\mathcal{M}}\right\}} d t\right)=\int_{0}^{\tau} \mathbb{P}\left(\sigma_{\mathcal{L}}-\sigma_{\mathcal{M}}<t\right) d t
$$

One might then compute $\mathbb{E}\left[\left(\sigma_{\mathcal{M}}+\tau-\sigma_{\mathcal{L}}\right)^{+}\right]$in a similar manner as Bérenguer $\&$ al. (2003) for the univariate case: the method relies on the computation of the survival function of $\sigma_{\mathcal{L}}-\sigma_{\mathcal{M}}$ (the distribution of which has a non zero mass at 0 because $\left.\mathbb{P}\left(\sigma_{\mathcal{L}}-\sigma_{\mathcal{M}}=0\right)>0\right)$ and implies some double differentiation of the survival function of the couple $\left(\sigma_{\mathcal{M}}, \sigma_{\mathcal{L}}\right)$. We however have better use here different arguments: noting that

$$
\left(\sigma_{\mathcal{M}}+\tau-\sigma_{\mathcal{L}}\right)^{+}=\left(\sigma_{\mathcal{L}}-\sigma_{\mathcal{M}}-\tau\right)^{+}+\sigma_{\mathcal{M}}+\tau-\sigma_{\mathcal{L}}
$$

and setting

$$
\begin{aligned}
g_{\mathcal{L}}(\mathcal{M}) & =\mathbb{E}\left[\left(\sigma_{\mathcal{L}}-\sigma_{\mathcal{M}}-\tau\right)^{+}\right] \\
h(\mathcal{M}) & =\mathbb{E}\left(\sigma_{\mathcal{M}}\right)
\end{aligned}
$$

for all $\mathcal{M} \supseteq \mathcal{L}$, one gets:

$$
\mathbb{E}\left[\left(\sigma_{\mathcal{M}}+\tau-\sigma_{\mathcal{L}}\right)^{+}\right]=g_{\mathcal{L}}(\mathcal{M})+h(\mathcal{M})+\tau-h(\mathcal{L})
$$

This provides the following expression for $C_{\infty}$ :

$$
C_{\infty}=C_{2}+\frac{C_{1}+C_{2}\left(g_{\mathcal{L}}(\mathcal{M})-h(\mathcal{L})\right)}{\tau+h(\mathcal{M})},
$$


where the different terms are now computable without too much technicality, as we next show.

Setting $\mathcal{L}-x=\left\{\left(y_{1}-x_{1}, y_{2}-x_{2}\right) \mid\left(y_{1}, y_{2}\right) \in \mathcal{L}\right\}$ for all $x=\left(x_{1}, x_{2}\right) \notin \mathcal{L}$ and

$$
\begin{aligned}
G_{t}(\mathcal{M}) & =\mathbb{P}\left(X_{t} \in \mathcal{M}\right)=\iint_{\mathcal{M}} \mathbb{P}_{X_{t}}\left(d x_{1}, d x_{2}\right), \\
\bar{G}_{t}(\mathcal{M}) & =\mathbb{P}\left(X_{t} \notin \mathcal{M}\right)=1-G_{t}(\mathcal{M})
\end{aligned}
$$

for all $t \geq 0$, we get the following result.

Proposition 3 Under assumption $\mathcal{H}$, we have

$$
\begin{aligned}
h(\mathcal{M}) & =\int_{0}^{\infty} \bar{G}_{t}(\mathcal{M}) d t \\
g_{\mathcal{L}}(\mathcal{M}) & =\int_{0}^{\infty} \iint_{\mathcal{M}} \bar{G}_{\tau}(\mathcal{L}-x) \mathbb{P}_{X_{v}}\left(d x_{1}, d x_{2}\right) d v
\end{aligned}
$$

where $\mathbb{P}_{X_{t}}$ stands for the probability distribution of $X_{t}$.

Proof. Since $\mathcal{M}$ is a closed upper set and $\left(X_{t}\right)_{t \geq 0}$ is a non-decreasing process, we have $\left\{\sigma_{\mathcal{M}}>t\right\}=\left\{X_{t} \notin \mathcal{M}\right\}$, from where we derive:

$$
h(\mathcal{M})=\int_{0}^{\infty} \mathbb{P}\left(\sigma_{\mathcal{M}}>t\right) d t=\int_{0}^{\infty} \mathbb{P}\left(X_{t} \notin \mathcal{M}\right)=\int_{0}^{\infty} \bar{G}_{t}(\mathcal{M}) d t
$$

As for the second expression, noting that

$$
g_{\mathcal{L}}(\mathcal{M})=\mathbb{E}\left(\int_{0}^{+\infty} \mathbf{1}_{\left\{\sigma_{\mathcal{L}}-\sigma_{\mathcal{M}}-\tau>u\right\}} d u\right)
$$

and setting $v=u+\sigma_{\mathcal{M}}$, we get:

$$
g_{\mathcal{L}}(\mathcal{M})=\mathbb{E}\left(\int_{0}^{\infty} \mathbf{1}_{\left\{\sigma_{\mathcal{M}} \leq v<\sigma_{\mathcal{L}}-\tau\right\}} d v\right)=\int_{0}^{\infty} \mathbb{P}\left[\sigma_{\mathcal{M}} \leq v<\sigma_{\mathcal{L}}-\tau\right] d v
$$

with

$$
\begin{aligned}
\mathbb{P}\left[\sigma_{\mathcal{M}} \leq v<\sigma_{\mathcal{L}}-\tau\right] & =\mathbb{P}\left[X_{v} \in \mathcal{M}, X_{v+\tau} \notin \mathcal{L}\right] \\
& =\mathbb{E}\left[\mathbf{1}_{\left\{X_{v} \in \mathcal{M}\right\}} \mathbb{E}\left(\mathbf{1}_{\left\{X_{v+\tau} \notin \mathcal{L}\right\}} \mid \mathcal{F}\left(X_{v}\right)\right)\right]
\end{aligned}
$$


where $\mathcal{F}\left(X_{v}\right)$ is the $\sigma$-algebra generated by $\left(X_{s}\right)_{0 \leq s \leq v}$. Using the Markov property at time $v$ and the homogeneous and independent increments of $\left(X_{t}\right)_{t \geq 0}$, we derive:

$$
\begin{aligned}
\mathbb{P}\left[\sigma_{\mathcal{M}} \leq v<\sigma_{\mathcal{L}}-\tau\right] & =\mathbb{E}\left[\mathbf{1}_{\left\{X_{v} \in \mathcal{M}\right\}} \mathbb{E}\left(\mathbf{1}_{\left\{X_{v+\tau}-X_{v} \notin \mathcal{L}-X_{v}\right\}} \mid X_{v}\right)\right] \\
& =\mathbb{E}\left[\mathbf{1}_{\left\{X_{v} \in \mathcal{M}\right\}} \bar{G}_{\tau}\left(\mathcal{L}-X_{v}\right)\right]
\end{aligned}
$$

and the result.

All involved quantities in $C_{\infty}$ only depend on the joint distribution of $X_{t}=\left(X_{t}^{(1)}, X_{2}^{(2)}\right)$ and on $\bar{G}_{t}$. The function $\bar{G}_{t}$ may easily be computed, as soon as the joint distribution of $X_{t}=\left(X_{t}^{(1)}, X_{2}^{(2)}\right)$ is available (which is not always the case for a bivariate subordinator). For instance, considering the three special cases of Subsection 2.1 and similar shapes for both $\mathcal{M}$ and $\mathcal{L}$, we get:

Case 1 For $\mathcal{M}=\mathbb{R}_{+}^{2} \backslash\left[0, M_{1}\left[\times\left[0, M_{2}\left[\right.\right.\right.\right.$ with $0<M_{1} \leq L_{1}$ and $0<M_{2} \leq L_{2}$ :

$$
\bar{G}_{t}(\mathcal{M})=\mathbb{P}\left(X_{t}^{(1)}<M_{1}, X_{t}^{(2)}<M_{2}\right)=\iint_{\left[0, M_{1}\left[\times\left[0, M_{2}[\right.\right.\right.} \mathbb{P}_{X_{v}}\left(d x_{1}, d x_{2}\right),
$$

Case 2 For $\mathcal{M}=\left[M_{1}, \infty\left[\times\left[M_{2}, \infty\left[\right.\right.\right.\right.$ with $0<M_{1} \leq L_{1}$ and $0<M_{2} \leq L_{2}$ :

$$
\bar{G}_{t}(\mathcal{M})=1-\mathbb{P}\left(X_{t}^{(1)} \geq M_{1}, X_{t}^{(2)} \geq M_{2}\right)=1-\iint_{\left[M_{1},+\infty\left[\times\left[M_{2},+\infty[\right.\right.\right.} \mathbb{P}_{X_{v}}\left(d x_{1}, d x_{2}\right),
$$

Case 3 For $\mathcal{M}=\left\{\left(x_{1}, x_{2}\right) \in \mathbb{R}_{+}^{2} \mid x_{1}+x_{2} \geq M\right\}$ with $0<M \leq L$ :

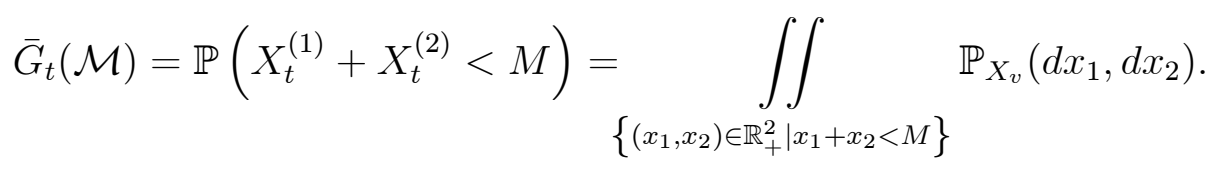

Using similar methods as for $C_{\infty}$, the asymptotic availability may be proved to exist almost surely and to be equal to the mean up time on a cycle divided by the mean cycle length: 


$$
A_{\infty}=\frac{\mathbb{E}\left(\sigma_{\mathcal{L}}\right)-\mathbb{E}\left[\left(\sigma_{\mathcal{L}}-\sigma_{\mathcal{M}}-\tau\right)^{+}\right]}{\tau+\mathbb{E}\left(\sigma_{\mathcal{M}}\right)}=\frac{h(\mathcal{L})-g_{\mathcal{L}}(\mathcal{M})}{\tau+h(\mathcal{M})},
$$

which involves the same quantities as $C_{\infty}$, and can consequently be computed at the same time.

As particular cases:

- For $\mathcal{M}=\mathcal{L}$ (unmaintained case), noting that $g_{\mathcal{L}}(\mathcal{L})=0$, we get:

$$
C_{\infty}^{(i n i)}=\frac{C_{1}+C_{2} \tau}{\tau+\mathbb{E}\left(\sigma_{\mathcal{L}}\right)} \text { and } A_{\infty}^{(i n i)}=\frac{\mathbb{E}\left(\sigma_{\mathcal{L}}\right)}{\tau+\mathbb{E}_{\mathcal{L}}\left(\sigma_{\mathcal{L}}\right)}
$$

- For $\mathcal{M}=\mathbb{R}_{+}^{2}$ (periodic replacements), we get:

$$
C_{\infty}^{(P R)}=\frac{C_{1}+C_{2} \mathbb{E}\left[\left(\tau-\sigma_{\mathcal{L}}\right)^{+}\right]}{\tau}
$$

and

$$
A_{\infty}^{(P R)}=\frac{\mathbb{E}\left(\sigma_{\mathcal{L}}\right)-\mathbb{E}\left[\left(\sigma_{\mathcal{L}}-\tau\right)^{+}\right]}{\tau}=\frac{\mathbb{E}\left[\min \left(\sigma_{\mathcal{L}}, \tau\right)\right]}{\tau}
$$

\subsection{Some comparison results}

We here provide a few comparison results between $C_{\infty}, C_{\infty}^{(P R)}$ and $C_{\infty}^{(i n i)}$.

Proposition 4 If $\frac{C_{1}}{C_{2}} \geq \mathbb{E}\left[\min \left(\tau, \sigma_{\mathcal{L}}\right)\right]$, then $C_{\infty} \leq C_{\infty}^{(P R)}$ (whatever $\mathcal{M}$ is) and the PM policy is better than a simple periodic replacement policy.

Proof. Noting that

$$
C_{\infty}^{(P R)}=C_{2}+\frac{C_{1}-C_{2}\left(\tau-\mathbb{E}\left[\left(\tau-\sigma_{\mathcal{L}}\right)^{+}\right]\right)}{\tau}=C_{2}+\frac{C_{1}-C_{2} \mathbb{E}\left[\min \left(\tau, \sigma_{\mathcal{L}}\right)\right]}{\tau}
$$

and using (4), the sign of $C_{\infty}-C_{\infty}^{(P R)}$ is the same as the sign of

$$
\begin{aligned}
& \tau\left[C_{1}+C_{2}\left(g_{\mathcal{L}}(\mathcal{M})-h(\mathcal{L})\right)\right]-\left[C_{1}-C_{2} \mathbb{E}\left[\min \left(\tau, \sigma_{\mathcal{L}}\right)\right]\right][\tau+h(\mathcal{M})] \\
& =-\left[C_{1}-C_{2} \mathbb{E}\left[\min \left(\tau, \sigma_{\mathcal{L}}\right)\right]\right] h(\mathcal{M})+\tau C_{2}\left(g_{\mathcal{L}}(\mathcal{M})-\mathbb{E}\left[\left(\sigma_{\mathcal{L}}-\tau\right)^{+}\right]\right)
\end{aligned}
$$


where $g_{\mathcal{L}}(\mathcal{M})-\mathbb{E}\left[\left(\sigma_{\mathcal{L}}-\tau\right)^{+}\right]=\mathbb{E}\left[\left(\sigma_{\mathcal{L}}-\sigma_{\mathcal{M}}-\tau\right)^{+}\right]-\mathbb{E}\left[\left(\sigma_{\mathcal{L}}-\tau\right)^{+}\right] \leq 0$, which provides the result.

As a special case $(\mathcal{M}=\mathcal{L})$, this result shows that if $\frac{C_{1}}{C_{2}} \geq \mathbb{E}\left[\min \left(\tau, \sigma_{\mathcal{L}}\right)\right]$, then $C_{\infty}^{(i n i)} \leq C_{\infty}^{(P R)}$.

Proposition 5 If $\frac{C_{1}}{C_{2}} \geq \mathbb{E}\left(\sigma_{\mathcal{L}}\right)$, then $C_{\infty} \geq C_{\infty}^{(i n i)}$ and the best is not to use the PM policy, namely call for the repair team only at $\sigma_{\mathcal{L}}$.

Proof. Starting from (4) and from

$$
C_{\infty}^{(i n i)}=C_{2}+\frac{C_{1}-C_{2} h(\mathcal{L})}{\tau+h(\mathcal{L})},
$$

the sign of $C_{\infty}-C_{\infty}^{(i n i)}$ is the same as the sign of

$$
\begin{aligned}
& {\left[C_{1}+C_{2}\left(g_{\mathcal{L}}(\mathcal{M})-h(\mathcal{L})\right)\right][\tau+h(\mathcal{L})]-\left[C_{1}-C_{2} h(\mathcal{L})\right][\tau+h(\mathcal{M})]} \\
& =\left(C_{1}-C_{2} h(\mathcal{L})\right)[h(\mathcal{L})-h(\mathcal{M})]+C_{2} g_{\mathcal{L}}(\mathcal{M})[\tau+h(\mathcal{L})]
\end{aligned}
$$

where $h(\mathcal{L})-h(\mathcal{M}) \geq 0$, which allows to conclude.

As a consequence from the previous results, if $\frac{C_{1}}{C_{2}} \geq \mathbb{E}\left(\sigma_{\mathcal{L}}\right)$, then $C_{\infty}^{(i n i)} \leq$ $C_{\infty} \leq C_{\infty}^{(P R)}$. Also, the only situation where the PM policy can be interesting (namely s.t. $C_{\infty} \leq C_{\infty}^{(i n i)}$ ) is the case where $\frac{C_{1}}{C_{2}}<\mathbb{E}\left(\sigma_{\mathcal{L}}\right)$.

\subsection{Influence of the delay time $\tau$ on $C_{\infty}$}

Though the delay time $\tau$ is generally fixed by the application context (and stands for the time required by the repair team to be ready to operate), we here consider that $\tau$ may vary, to better understand its influence both on the maintained and unmaintained system. We write $C_{\infty}(\tau)$ instead of $C_{\infty}$ in all the subsection.

Proposition 6 1. We have $\lim _{\tau \rightarrow+\infty} C_{\infty}(\tau)=C_{2}$. 
2. If $\mathbb{E}\left(\sigma_{\mathcal{L}}\right)<\frac{C_{1}}{C_{2}}$, the cost function $C_{\infty}(\tau)$ is decreasing with respect of $\tau$, whatever $\mathcal{M}$ is.

3. If $\mathbb{E}\left(\sigma_{\mathcal{L}}\right) \geq \frac{C_{1}}{C_{2}}$, assuming $\mathcal{L} \varsubsetneqq \mathcal{M}$ and noting that $\mathbb{P}\left(\sigma_{\mathcal{L}}=\sigma_{\mathcal{M}}\right) \mathbb{E}\left(\sigma_{\mathcal{M}}\right)<$ $\mathbb{E}\left(\sigma_{\mathcal{L}}\right)$, we have the following dichotomy:

- if $\frac{C_{1}}{C_{2}} \leq \mathbb{P}\left(\sigma_{\mathcal{L}}=\sigma_{\mathcal{M}}\right) \mathbb{E}\left(\sigma_{\mathcal{M}}\right)$ : the cost function $C_{\infty}(\tau)$ is non decreasing with respect of $\tau$,

- if $\mathbb{P}\left(\sigma_{\mathcal{L}}=\sigma_{\mathcal{M}}\right) \mathbb{E}\left(\sigma_{\mathcal{M}}\right)<\frac{C_{1}}{C_{2}} \leq \mathbb{E}\left(\sigma_{\mathcal{L}}\right)$ : the cost function $C_{\infty}(\tau)$ admits a unique minimum at some $\tau_{\mathcal{M}}$ (with $\left.0<\tau_{\mathcal{M}}<+\infty\right)$ such that:

$$
\int_{0}^{\tau_{\mathcal{M}}} \mathbb{P}\left(t<\sigma_{\mathcal{L}}-\sigma_{\mathcal{M}} \leq \tau_{\mathcal{M}}\right) d t+\mathbb{P}\left(\sigma_{\mathcal{L}}-\sigma_{\mathcal{M}} \leq \tau_{\mathcal{M}}\right) \mathbb{E}\left(\sigma_{\mathcal{M}}\right)-\frac{C_{1}}{C_{2}}=0
$$

Proof. Using (2) and (3), we may write:

$$
C_{\infty}(\tau)=\frac{C_{1}+C_{2} \int_{0}^{\tau} \mathbb{P}\left(\sigma_{\mathcal{L}}-\sigma_{\mathcal{M}} \leq t\right) d t}{\tau+\mathbb{E}\left(\sigma_{\mathcal{M}}\right)}
$$

We derive:

$$
\lim _{\tau \rightarrow+\infty} C_{\infty}(\tau)=C_{2} \times \lim _{\tau \rightarrow+\infty} \frac{\int_{0}^{\tau} \mathbb{P}\left(\sigma_{\mathcal{L}}-\sigma_{\mathcal{M}} \leq t\right) d t}{\int_{0}^{\tau} 1 d t}=C_{2},
$$

using L'hôpital's rule, which proves the first point.

As for the second and third points, starting from (7), the sign of $\frac{\partial}{\partial \tau} C_{\infty}(\tau)$ is the same as the sign of

$$
\begin{aligned}
H(\tau) & =\mathbb{P}\left(\sigma_{\mathcal{L}}-\sigma_{\mathcal{M}} \leq \tau\right)\left(\tau+\mathbb{E}\left(\sigma_{\mathcal{M}}\right)\right)-\left(\frac{C_{1}}{C_{2}}+\int_{0}^{\tau} \mathbb{P}\left(\sigma_{\mathcal{L}}-\sigma_{\mathcal{M}} \leq t\right) d t\right) \\
& =\int_{0}^{\tau} \mathbb{P}\left(t<\sigma_{\mathcal{L}}-\sigma_{\mathcal{M}} \leq \tau\right) d t+\mathbb{P}\left(\sigma_{\mathcal{L}}-\sigma_{\mathcal{M}} \leq \tau\right) \mathbb{E}\left(\sigma_{\mathcal{M}}\right)-\frac{C_{1}}{C_{2}}
\end{aligned}
$$

which is non decreasing in $\tau$ (constant in case $\mathcal{M}=\mathcal{L})$. Besides:

$$
\lim _{\tau \rightarrow \infty} H(\tau)=\mathbb{E}\left(\sigma_{\mathcal{L}}-\sigma_{\mathcal{M}}\right)+\mathbb{E}\left(\sigma_{\mathcal{M}}\right)-\frac{C_{1}}{C_{2}}=\mathbb{E}\left(\sigma_{\mathcal{L}}\right)-\frac{C_{1}}{C_{2}}
$$


and $H\left(0^{+}\right)=\mathbb{P}\left(\sigma_{\mathcal{L}}=\sigma_{\mathcal{M}}\right) \mathbb{E}\left(\sigma_{\mathcal{M}}\right)-\frac{C_{1}}{C_{2}}$. This easily provides the results.

This proposition shows that the behavior of the cost function with respect of $\tau$ may be quite different according to the case. As an example, in case of a high replacement cost $\left(\mathbb{E}\left(\sigma_{\mathcal{L}}\right)<\frac{C_{1}}{C_{2}}\right)$, we can see that, from a cost point of view, the best is not to ever repair the system. Even if some benefit for uptime were considered in the cost function, such a result would still be valid in case of too high a replacement cost. In this situation, the system does not bring any profit, with or without preventive maintenance. If there is still some interest in the functioning of the system (which may be some client satisfaction e.g.), one should then control another reliability indicator, such as the system availability. It is easy to check that the system availability is always decreasing with $\tau$. The optimal value of $\tau$ may then be provided by optimizing the cost function under some availability constraint, namely chose the largest $\tau$ which meets with the availability constraint. As an alternative, one may also optimize the availability under some cost constraint, namely chose the shortest $\tau$ which meets with the cost constraint.

More generally, from a cost point of view, one can observe that it is not necessary mandatory that the repair team arrives as soon as possible (with the shortest $\tau$ ) and some added delay in the repair may improve the cost function. However, such an added delay always decreases the availability, and it should then be controlled.

The probability $\mathbb{P}\left(\sigma_{\mathcal{L}}=\sigma_{\mathcal{M}}\right)$ might be computed using martingale technics as in Bertoin (1996). It however is rather technical and does not bring much to the present study. We consequently do not go any further into this direction.

Note that the present results do not depend on the dimension and would be valid in the univariate or multivariate cases. 


\section{Numerical experiments}

In order to illustrate the results, a bivariate Gamma process is used, which is constructed by trivariate reduction, as proposed by Cherian (1941) in the case of bivariate Gamma random vectors.

\subsection{A bivariate Gamma process}

Let us first recall that an univariate Gamma process with parameters $(a, b)$ (where $a, b>0$ ) is a subordinator such that for every $t \geq 0$, the random variable $Y_{t}$ is Gamma distributed $\Gamma(a t, b)$ with probability distribution function (p.d.f.):

$$
f_{a t, b}(x)=\frac{1}{\Gamma(a t)} b^{a t} e^{-b x} x^{a t-1} 1_{\{x>0\}} .
$$

We only envision the case $b=1$ in the following (no restriction) and we set $f_{a t, b}=f_{a t}$. The corresponding cumulative distribution function (c.d.f.) and survival function are denoted by $F_{a t}$ and $\bar{F}_{a t}$, respectively, with $\bar{F}_{a t}=1-F_{a t}$.

Starting from three independent univariate Gamma processes $\left(Y_{t}^{(i)}\right)_{t \geq 0}$ with parameters $\left(\alpha_{i}, 1\right)$ for $i=1,2,3$ (where $\alpha_{1}, \alpha_{2}, \alpha_{3}>0$ ), we set

$$
X_{t}^{(1)}=Y_{t}^{(1)}+Y_{t}^{(3)} \text { and } X_{t}^{(2)}=Y_{t}^{(2)}+Y_{t}^{(3)}
$$

The process $\left(X_{t}\right)_{t \geq 0}=\left(X_{t}^{(1)}, X_{t}^{(2)}\right)_{t \geq 0}$ then is a bivariate subordinator with Gamma marginal processes and marginal parameters $\left(a_{i}, 1\right)$ where $a_{i}=\alpha_{i}+$ $\alpha_{3}$ for $i=1,2$. The linear correlation coefficient between the two random variables $X_{t}^{(1)}$ and $X_{t}^{(2)}$ is independent of $t$ and given by

$$
\rho=\frac{\alpha_{3}}{\sqrt{a_{1} a_{2}}}
$$

We consequently have $\alpha_{1}=a_{1}-\rho \sqrt{a_{1} a_{2}}, \alpha_{2}=a_{2}-\rho \sqrt{a_{1} a_{2}}$ and $\alpha_{3}=$ $\rho \sqrt{a_{1} a_{2}}$, with $0 \leq \rho \leq \rho_{\max }=\min \left(\sqrt{\frac{a_{1}}{a_{2}}}, \sqrt{\frac{a_{2}}{a_{1}}}\right)$, see Devroye (1986) e.g.. 
Two equivalent alternate parameterizations hence are available for $\left(X_{t}\right)_{t \geq 0}$ : either $\left(\alpha_{1}, \alpha_{2}, \alpha_{3}\right)$ or $\left(a_{1}, a_{2}, \rho\right)$. Besides, all the dependence between the marginal processes is contained in the linear correlation coefficient $\rho$.

The joint p.d.f. of the random vector $X_{t}=\left(X_{t}^{(1)}, X_{t}^{(2)}\right)$ is provided by:

$$
f_{X_{t}}\left(x_{1}, x_{2}\right)=\int_{0}^{+\infty} f_{\alpha_{1} t}\left(x_{1}-x_{3}\right) f_{\alpha_{2} t}\left(x_{2}-x_{3}\right) f_{\alpha_{3} t}\left(x_{3}\right) d x_{3} .
$$

The corresponding c.d.f. and survival function are provided by:

$$
\begin{aligned}
F_{X_{t}}\left(x_{1}, x_{2}\right) & =\mathbb{P}\left(X_{t}^{(1)} \leq x_{1}, X_{t}^{(2)} \leq x_{2}\right) \\
& =\int_{0}^{+\infty} F_{\alpha_{1} t}\left(x_{1}-x_{3}\right) F_{\alpha_{2} t}\left(x_{2}-x_{3}\right) f_{\alpha_{3} t}\left(x_{3}\right) d x_{3}
\end{aligned}
$$

and

$$
\begin{aligned}
\bar{F}_{X_{t}}\left(x_{1}, x_{2}\right) & =\mathbb{P}\left(X_{t}^{(1)}>x_{1}, X_{t}^{(2)}>x_{2}\right) \\
& =\int_{0}^{+\infty} \bar{F}_{\alpha_{1} t}\left(x_{1}-x_{3}\right) \bar{F}_{\alpha_{2} t}\left(x_{2}-x_{3}\right) f_{\alpha_{3} t}\left(x_{3}\right) d x_{3}
\end{aligned}
$$

for all $t, x_{1}, x_{2} \in \mathbb{R}_{+}$.

Considering the three special cases of Subsections 2.1 and 3.1, the functions $h(\mathcal{M})$ and $g_{\mathcal{L}}(\mathcal{M})$ which appear in $C_{\infty}$ and $A_{\infty}$ are computed via the following.

Case $1\left(\mathcal{L}=\mathbb{R}_{+}^{2} \backslash\left[0, L_{1}\left[\times\left[0, L_{2}[)\right.\right.\right.\right.$ We have $\bar{G}_{t}(\mathcal{M})=F_{X_{t}}\left(M_{1}, M_{2}\right)$ and

$$
h(\mathcal{M})=\iint_{\mathbb{R}_{+}^{2}} F_{\alpha_{1} t}\left(M_{1}-x_{3}\right) F_{\alpha_{2} t}\left(M_{2}-x_{3}\right) f_{\alpha_{3} t}\left(x_{3}\right) d x_{3} d t .
$$

Writing $\iint_{\mathcal{M}}=\iint_{\mathbb{R}_{+}^{2}}-\iint_{\left[0, M_{1}\left[\times\left[0, M_{2}[\right.\right.\right.}$ and using $F_{\alpha_{i} \tau} * f_{\alpha_{i} t}=F_{\alpha_{i}(\tau+t)}$, 
we also get:

$$
\begin{aligned}
g_{\mathcal{L}}(\mathcal{M}) & =\iint_{\mathbb{R}_{+}^{2}} F_{\alpha_{1}(\tau+t)}\left(L_{1}-x_{3}\right) F_{\alpha_{2}(\tau+t)}\left(L_{2}-x_{3}\right) f_{\alpha_{3}(\tau+t)}\left(x_{3}\right) d x_{3} d t \\
& -\iiint_{\mathbb{R}_{+}^{3}} f_{\alpha_{3} \tau}\left(x_{3}\right) f_{\alpha_{3} t}\left(z_{3}\right) \\
& \times\left[\iint_{\left[0, M_{1}\left[\times\left[0, M_{2}[\right.\right.\right.} F_{\alpha_{1} \tau}\left(L_{1}-x_{1}-x_{3}\right) F_{\alpha_{2} \tau}\left(L_{2}-x_{2}-x_{3}\right)\right. \\
\times & \left.f_{\alpha_{1} t}\left(x_{1}-z_{3}\right) f_{\alpha_{2} t}\left(x_{2}-z_{3}\right) d x_{1} d x_{2}\right] d t d x_{3} d z_{3} .
\end{aligned}
$$

Case $2\left(\mathcal{L}=\left[L_{1}, \infty\left[\times\left[L_{2}, \infty[)\right.\right.\right.\right.$ We have :

$$
\begin{aligned}
\bar{G}_{t}(\mathcal{M}) & =1-\bar{F}_{X_{t}}\left(M_{1}, M_{2}\right)=F_{a_{1} t}\left(M_{1}\right)+F_{a_{2} t}\left(M_{2}\right)-F_{X_{t}}\left(M_{1}, M_{2}\right), \\
h(\mathcal{M}) & =\int_{0}^{\infty} F_{a_{1} t}\left(M_{1}\right) d t+\int_{0}^{\infty} F_{a_{2} t}\left(M_{2}\right) d t \\
& -\iint_{\mathbb{R}_{+}^{2}} F_{\alpha_{1} t}\left(M_{1}-x_{3}\right) F_{\alpha_{2} t}\left(M_{2}-x_{3}\right) f_{\alpha_{3} t}\left(x_{3}\right) d x_{3} d t,
\end{aligned}
$$

and

$$
\begin{aligned}
g_{\mathcal{L}}(\mathcal{M})=\iint_{\left[M_{1}, \infty\left[\times\left[M_{2}, \infty[\right.\right.\right.} & {\left[F_{a_{1} \tau}\left(L_{1}-x_{1}\right)+F_{a_{2} \tau}\left(L_{2}-x_{2}\right)\right.} \\
& \left.-\int_{0}^{+\infty} F_{\alpha_{1} \tau}\left(L_{1}-x_{1}-u\right) F_{\alpha_{2} \tau}\left(L_{2}-x_{2}-u\right) f_{\alpha_{3} \tau}(u) d u\right] \\
& \times\left(\iint_{\mathbb{R}_{+}^{2}} f_{\alpha_{1} v}\left(x_{1}-w\right) f_{\alpha_{2} v}\left(x_{2}-w\right) f_{\alpha_{3} v}(w) d v d w\right) d x_{1} d x_{2}
\end{aligned}
$$

Case $3\left(\mathcal{L}=\left\{\left(x_{1}, x_{2}\right) \in \mathbb{R}_{+}^{2} \mid x_{1}+x_{2} \geq L\right\}\right)$ We have:

$$
\begin{aligned}
& \bar{G}_{t}(\mathcal{M})=\mathbb{P}\left(Y_{t}^{(1)}+Y_{t}^{(2)}+2 Y_{t}^{(3)}<M\right)=\int_{0}^{+\infty} F_{\left(\alpha_{1}+\alpha_{2}\right) t}(M-2 y) f_{\alpha_{3} t}(y) d y \\
& \text { since } Y_{t}^{(1)}+Y_{t}^{(2)} \text { is Gamma distributed with parameters }\left(\left(\alpha_{1}+\alpha_{2}\right) t, 1\right) .
\end{aligned}
$$




\begin{tabular}{lccc}
\hline & Analytical formula & MC simulations & MC 95\% confidence interval \\
\hline$C_{\infty}$ & 154.21612 & 154.38232 & {$[152.79936 ; 155.96529]$} \\
$A_{\infty}$ & 0.87203 & 0.87245 & {$[0.85885 ; 0.88604]$} \\
\hline
\end{tabular}

Table 1: Comparison with MC simulations, Case 1 (series system)

Hence: $h(\mathcal{M})=\iint_{\mathbb{R}_{+}^{2}} F_{\left(\alpha_{1}+\alpha_{2}\right) t}(M-2 y) f_{\alpha_{3} t}(y) d t d y$ and

$$
\begin{aligned}
g_{\mathcal{L}}(\mathcal{M}) & =\iiint \int_{\mathbb{R}_{+}^{4}} f_{\alpha_{1} t}\left(x_{1}-x_{3}\right) f_{\alpha_{3} t}\left(x_{3}\right) f_{\alpha_{3} \tau}(y) \\
& \times\left(\int_{M-x_{1}}^{\infty} f_{\alpha_{2} t}\left(x_{2}-x_{3}\right) F_{\left(\alpha_{1}+\alpha_{2}\right) \tau}\left(L-x_{1}-x_{2}-2 y\right) d x_{2}\right) d t d x_{1} d x_{3} d y
\end{aligned}
$$

\subsection{Validation of the numerical results}

Both $C_{\infty}$ and $A_{\infty}$ are here computed on a few examples, via the previous analytical results and by Monte-Carlo (MC) simulations, with $10^{4}$ stories. For the MC results, the regenerative property of the system is exploited to derive some $95 \%$ confidence band for the results, see Asmussen (2003) or Eymard \& Mercier (2008) e.g.. We consider the three different cases for the shape of $(\mathcal{M}, \mathcal{L})$.

Case 1 We take $a_{1}=4, a_{2}=5, \rho=0.6708, \tau=0.1, M_{1}=3.4, M_{2}=2.4$, $L_{1}=3.5, L_{2}=2.5, C_{1}=100$ and $C_{2}=30$. The results are displayed in Table 1.

Case 2 We take $a_{1}=7, a_{2}=9, \rho=0.75, \tau=0.1, M_{1}=2.9, M_{2}=2.3$, $L_{1}=3.5, L_{2}=2.5, C_{1}=100$ and $C_{2}=30$. The results are displayed in Table 2.

Case 3 We take $a_{1}=4, a_{2}=9, \rho=0.4, \tau=0.1, M=2.4, L=3.5, C_{1}=3$ and $C_{2}=1$. The results are displayed in Table 3 . 


\begin{tabular}{lccc}
\hline & Analytical formula & MC simulations & MC 95\% confidence interval \\
\hline$C_{\infty}$ & 172.60371 & 171.04858 & {$[168.722395 ; 173.3732]$} \\
$A_{\infty}$ & 0.91734 & 0.917911 & {$[0.90132 ; 0.93450]$} \\
\hline
\end{tabular}

Table 2: Comparison with MC simulations, Case 2 (parallel system)

\begin{tabular}{cccc}
\hline & Analytical formula & MC simulations & MC 95\% confidence interval \\
\hline$C_{\infty}$ & 9.0611 & 9.0461 & {$[8.6960 ; 9.3961]$} \\
$A_{\infty}$ & 0.8750 & 0.8738 & {$[0.8407 ; 0.9069]$} \\
\hline
\end{tabular}

Table 3: Comparison with MC simulations, Case 3

In each case, the results by MC simulations and by the analytical formulae are coherent, which validate the method.

\subsection{Examples}

We now illustrate our results through different numerical experimentations. The parameters of the bivariate Gamma process and the shape of $(\mathcal{M}, \mathcal{L})$ are provided in Table 4 for each example.

Example 1 Two different values are considered for $C_{1}: C_{1}=0.198$ and $C_{1}=0.594$, and $C_{\infty}$ is plotted against the delay time $\tau$ in Figure 3 for both values. In the first case (Figure 3a), $C_{1}$ is such that $\frac{C_{1}}{C_{2}}<\mathbb{E}\left(\sigma_{\mathcal{L}}\right)$ and the cost function $C_{\infty}$ is minimum at $\tau_{0}^{\text {opt }} \simeq 0.0625$. In the second case (Figure $3 b$ ), we have $\frac{C_{1}}{C_{2}}>\mathbb{E}\left(\sigma_{\mathcal{L}}\right)$ and $C_{\infty}$ is decreasing with $\tau$. Such behaviors are coherent with the results of Proposition 6. In case $\frac{C_{1}}{C_{2}}>\mathbb{E}\left(\sigma_{\mathcal{L}}\right)$, the lowest cost is obtained for $\tau=\infty$ which means that, from a cost point of view, the best is not to ever repair the system. As explained in the lines following Proposition 6, one should then control the asymptotic availability, which is also decreasing in 


\begin{tabular}{lccccccccccc}
\hline & $a_{1}$ & $a_{2}$ & $\rho$ & $\tau$ & $\begin{array}{c}\text { shape of } \\
(\mathcal{M}, \mathcal{L})\end{array}$ & $\begin{array}{c}L_{1} \\
(\text { or } L)\end{array}$ & $\begin{array}{c}L_{2} \\
(\text { or } M)\end{array}$ & $M_{1}$ & $C_{1}$ & $C_{2}$ \\
\hline Ex. 1 & 4 & 9 & 0.5 & - & case 1 & 3.5 & 2.5 & 2.8 & 2 & - & 1 \\
Ex. 2 & 7 & 9 & 0.76 & 0.1 & case 2 & 3.5 & 2.5 & - & - & - & 1 \\
Ex. 3 & 4 & 9 & - & 0.1 & case 1 & 3.5 & 2.5 & 2.8 & 2 & 20 & - \\
& 7 & 9 & - & 0.1 & case 2 & 3.5 & 2.5 & 2.9 & 2.3 & 20 & - \\
Ex. 4 & 4 & - & - & 0.1 & case 3 & 3.5 & & 2.4 & & 1 & - \\
\hline
\end{tabular}

Table 4: Parameters and shapes of $(\mathcal{M}, \mathcal{L})$ for the different examples

$\tau$, see Figure 3c. Assume for instance that we have an availability constraint provided by $A_{\infty} \geq 0.9$ (to ensure client satifaction e.g.). The optimal value of $\tau$ which minimizes the cost function under this availability constraint then is the largest $\tau$ which fulfills this constraint, namely $\tau_{0} \simeq 0.075$.

Example 2 Two different values are considered for $C_{1}: C_{1}=0.15$ and $C_{1}=2$, and $C_{\infty}$ is plotted against $\left(M_{1}, M_{2}\right)$ in Figure 4 for both values. In the first case (Figure 4a), $C_{1}$ is such that $\frac{C_{1}}{C_{2}}<\mathbb{E}\left(\sigma_{\mathcal{L}}\right)$ and the cost is minimum at $\left(M_{1}^{\text {opt }}, M_{2}^{\text {opt }}\right) \simeq(2.8,1.8)$. In the second case, we have $C_{1}>C_{2} \mathbb{E}\left(\sigma_{\mathcal{L}}\right)$ (Figure $4 b)$ and the cost is minimum at $\left(L_{1}, L_{2}\right)$, which means that no PM policy is required. Such a behavior is coherent with the results of Proposition 5 .

Example 3 We take three different values for $C_{2}: C_{2}=4, C_{2}=20$ and $C_{2}=30$, and two different shapes for $(\mathcal{M}, \mathcal{L})$. The cost is plotted against the dependence (measured by $\rho$ ) in Figure 5 in all these cases. For the first shape of $(\mathcal{M}, \mathcal{L})$ (case 1, Figure 1a), we observe that the cost is decreasing with $\rho$ for the three values of $C_{1}$ (Figure $\left.5 a\right)$. For the second shape of $(\mathcal{M}, \mathcal{L})$ (case 


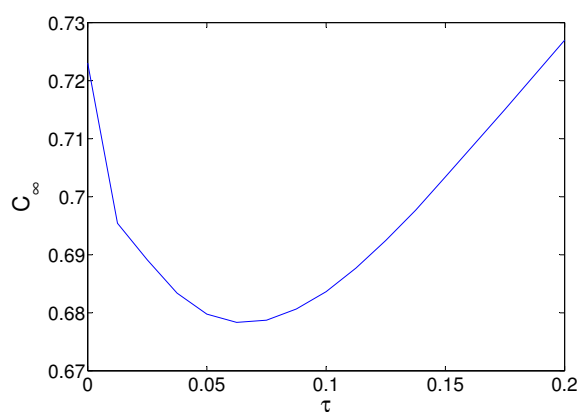

(a) $C_{\infty}$, case $\frac{C_{1}}{C_{2}}<\mathbb{E}\left(\sigma_{L}\right)$

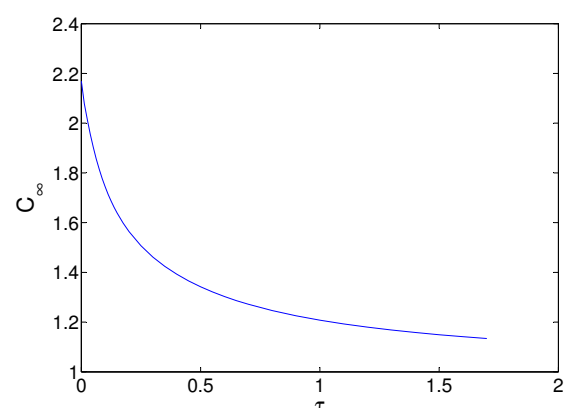

(b) $C_{\infty}$, case $\frac{C_{1}}{C_{2}}>\mathbb{E}\left(\sigma_{L}\right)$

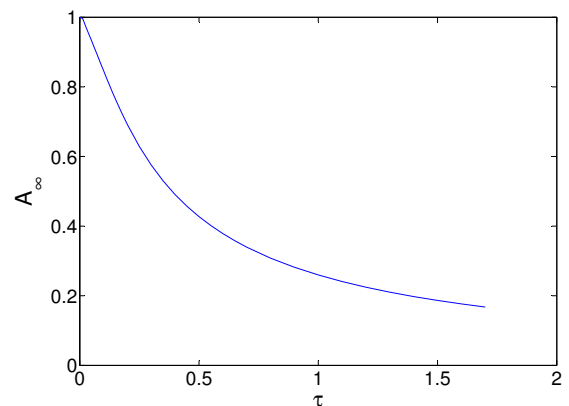

(c) $A_{\infty}$, case $\frac{C_{1}}{C_{2}}>\mathbb{E}\left(\sigma_{L}\right)$

Figure 3: $C_{\infty}$ and $A_{\infty}$ as a function of $\tau$, Example 1.

2, Figure 1b), the monotony is reversed (Figure 5b) and the cost is increasing with $\rho$.

Example 4 We here consider the third shape for $(\mathcal{M}, \mathcal{L})$ (case 3, Figure 1c) and the cost is plotted against $\rho$ for four different couples $\left(a_{2}, C_{2}\right)$ in Figure 6 , with $\left(a_{2}, C_{2}\right) \in\{(9,1),(9,10),(4,30),(4,7)\}$. According to these four cases, we can see that the cost may be increasing, decreasing, concave, convex with respect of $\rho$, so that nothing can be said about the behavior of the cost function with respect of the dependence. 


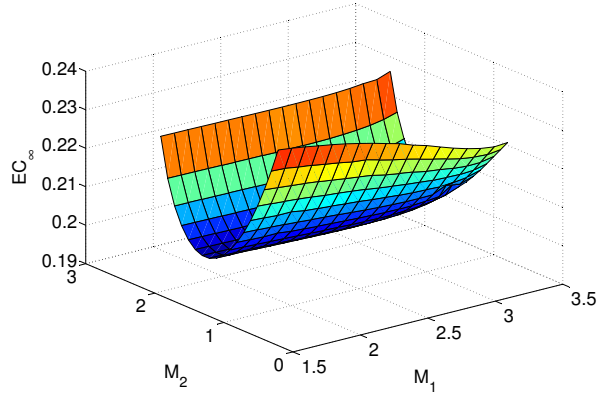

(a) Case $C_{1} / C_{2}<\mathbb{E}\left(\sigma_{L}\right)$

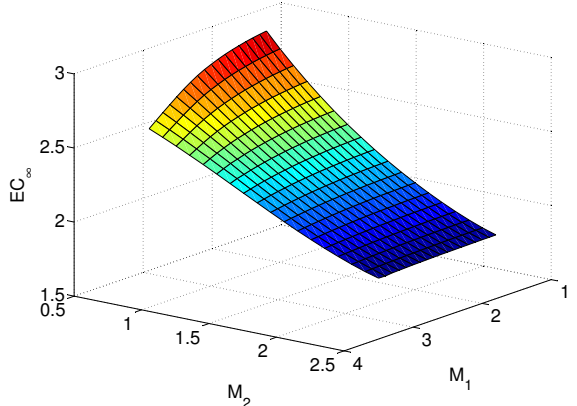

(b) Case $C_{1} / C_{2}>\mathbb{E}\left(\sigma_{L}\right)$

Figure 4: $C_{\infty}$ as a function of $\left(M_{1}, M_{2}\right)$, Example 2 .

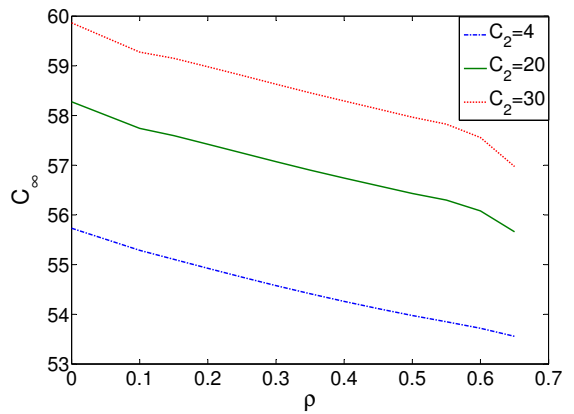

(a) Case 1 - the first shape

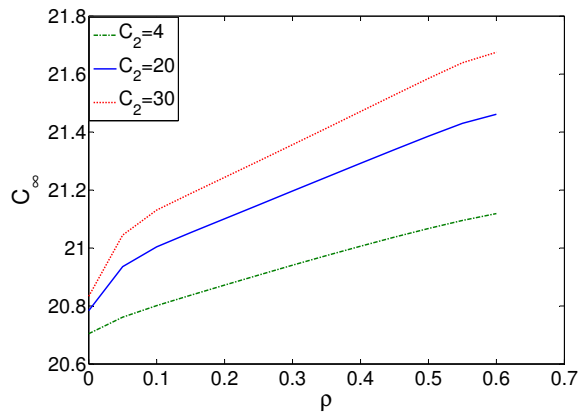

(b) Case 2 - the second shape

Figure 5: $C_{\infty}$ as a function of $\rho$, Example 3.

\section{Conclusion}

We here proposed a PM policy for a continuously monitored system modeled by a bivariate subordinator. The PM policy has been assessed through a cost function on an infinite horizon time. We studied some conditions under which the PM policy decreases the cost function when compared to a simple periodic replacement policy or to the unmaintained case. Though the delay time $\tau$ is generally fixed by the application context, we here studied its influence on the cost function. As a result, we have seen that, in case of high replacement costs, some added delay in the repair might improve the cost function. This 


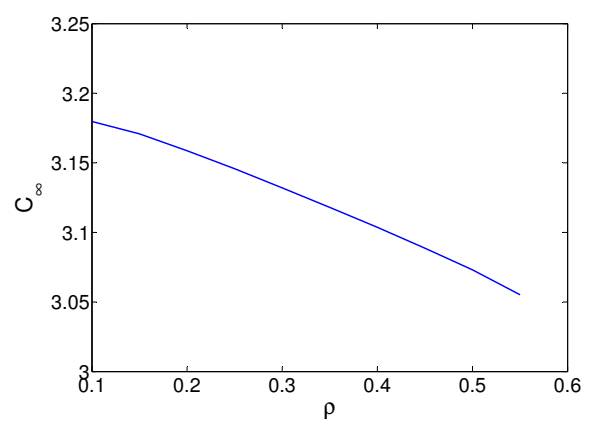

(a) $a_{2}=9, C_{2}=1$

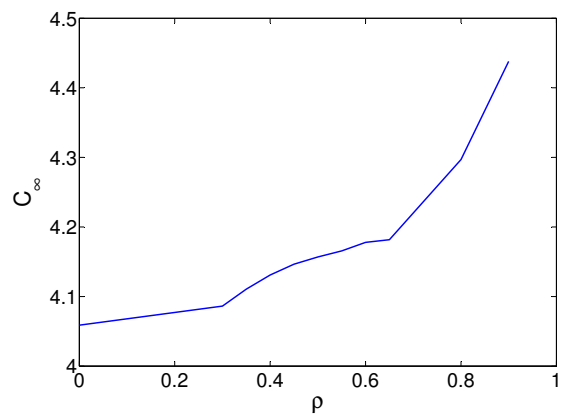

(c) $a_{2}=4, C_{2}=30$

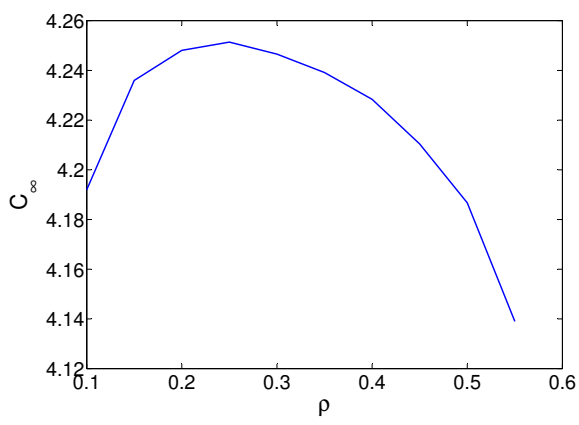

(b) $a_{2}=9, C_{2}=10$

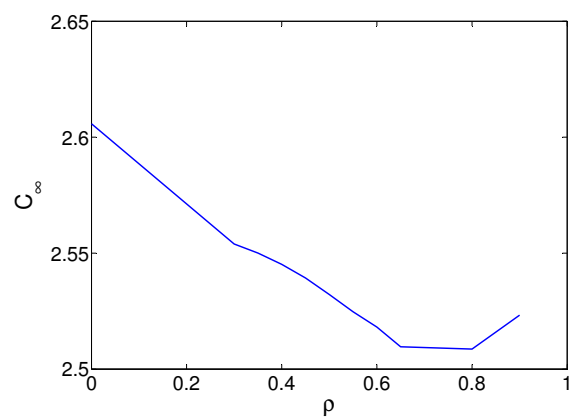

(d) $a_{2}=4, C_{2}=7$

Figure 6: $C_{\infty}$ as a function of $\rho$, Example 4, Case 3 - the third shape.

means that from a cost point of view, it is not necessary mandatory that the repair team arrives as soon as possible. However, such an added delay always decreases the availability, and it should then be controlled (or any other reliability indicator), in complement to the cost function.

As for the influence of the dependence between the two wear indicators on the cost function, we have not been able to study it from a theoretical point of view. We have however numerically observed that the cost function seemed to be monotonic with respect to the dependence for the first two shapes of failure regions considered in the paper (decreasing for the first shape and increasing for the second one). The proof of these monotonicity results remains a challenging open question. As for the third envisioned shape, the different examples show that the cost function is not monotonic with the 
dependence. The shape of the failure region hence has a clear influence on the eventual monotonicity of the cost function with respect to the dependence. According to the case, not taking into account the dependence between the wear indicators may hence lead to under- or over-estimate the cost function (see Figures 5 and 6), which may induce difficulties in an industrial context. Taking into account the dependence as in the present paper then is essential.

We here made the choice of studying asymptotic unitary indicators. Another possibility might have been to use transitory indicators such as pointwise availability and finite time cumulated cost. Indeed, using the regenerative structure of the maintained system, one should then be able to write renewal equations fullfilled by these transitory indicators, which might be solved numerically.

Acknowledgement 1 Hai Ha PHAM thanks the Conseil Régional d'Aquitaine (France) which supports her research work. Both authors thank the referee for his/her useful comments which allowed them to clarify some points of the paper and to improve its presentation.

\section{References}

Abdel-Hameed, M. (1975). A Gamma wear process. IEEE Trans. Reliability 24(2): 152-153.

Asmussen, S. (2003). Applied probability and queues. Applications of Mathematics 51. Springer-Verlag, New York, second edition.

Barlow, R. E. \& Proschan, F. (1965). Mathematical theory of reliability. Classics in Applied Mathematics 17, Society for Industrial and Applied Mathematics (SIAM), Philadelphia, PA. 
APPENDIX: NOTATIONS

\begin{tabular}{|c|c|}
\hline $\mathrm{PM}$ & Preventive Maintenance \\
\hline$\left(X_{t}\right)_{t \geq 0}=\left(X_{t}^{(1)}, X_{t}^{(2)}\right)_{t \geq 0}$ & Intrinsec system deterioration, bivariate subordinator \\
\hline $\mathcal{L}$ & Failure zone \\
\hline $\mathcal{M}$ & PM zone \\
\hline$\sigma_{\mathcal{L}}$ & Time to failure \\
\hline$\sigma_{\mathcal{M}}$ & Reaching time of PM zone \\
\hline$\tau$ & Deterministic delay time \\
\hline Upper sets $1^{s t}$ shape & $\mathbb{R}_{+}^{2} \backslash\left[0, M_{1}\left[\times\left[0, M_{2}[\right.\right.\right.$ \\
\hline Upper sets $2^{\text {nd }}$ shape & {$\left[M_{1}, \infty\left[\times\left[M_{2}, \infty[\right.\right.\right.$} \\
\hline Upper sets $3^{r d}$ shape & $\left\{\left(x_{1}, x_{2}\right) \in \mathbb{R}_{+}^{2}: x_{1}+x_{2} \geq M\right\}$ \\
\hline$L_{i}, i=1,2$ & Univariate failure threshold \\
\hline$\sigma_{L_{i}}^{(i)}, i=1,2$ & Univariate failure time \\
\hline$M_{i}, i=1,2$ & Univariate PM threshold \\
\hline$\sigma_{M_{i}}^{(i)}, i=1,2$ & Univariate reaching time of PM threshold \\
\hline$\left(Z_{t}\right)_{t \geq 0}$ & Regenerative process describing the system under PM \\
\hline$C_{1}$ & Restoration cost of the system \\
\hline$C_{2}$ & Unitary cost for down time \\
\hline$C_{\infty}$ & Asymptotic unitary cost \\
\hline$A_{\infty}$ & Asymptotic availability \\
\hline$C_{\infty}^{i n i}$ & Asymptotic unitary cost of the unmaintained system \\
\hline$A_{\infty}^{i n i}$ & Asymptotic availability of the unmaintained system \\
\hline$C_{\infty}^{P R}$ & Asymptotic unitary cost in case of periodic replacements \\
\hline$A_{\infty}^{P R}$ & Asymptotic availability in case of periodic replacements \\
\hline$G_{t}(\mathcal{M})$ & Probability of $X_{t}$ in $\mathcal{M}$ \\
\hline $\bar{G}_{t}(\mathcal{M})$ & Probability of $X_{t}$ outside $\mathcal{M}$ \\
\hline$h(\mathcal{M})$ & Mean of $\sigma_{\mathcal{M}}$ \\
\hline$g_{\mathcal{L}}(\mathcal{M})$ & Mean of $\left(\sigma_{\mathcal{L}}-\sigma_{\mathcal{M}}-\tau\right)^{+}$ \\
\hline
\end{tabular}




\begin{tabular}{|ll|}
\hline $\mathbb{P}_{X_{t}}\left(d x_{1}, d x_{2}\right)$ & joint distribution of $X_{t}=\left(X_{t}^{(1)}, X_{t}^{(2)}\right)$, general case \\
c.d.f. & cumulative distribution function \\
p.d.f. & probability distribution function \\
s.f. & survival function \\
r.v. & random variable \\
$f_{X_{t}}\left(x_{1}, x_{2}\right)$ & joint p.d.f. of $X_{t}=\left(X_{t}^{(1)}, X_{t}^{(2)}\right)$ \\
$F_{X_{t}}\left(x_{1}, x_{2}\right)$ & joint c.d.f. of $X_{t}=\left(X_{t}^{(1)}, X_{t}^{(2)}\right)$ \\
$\bar{F}_{X_{t}}\left(x_{1}, x_{2}\right)$ & joint s.f. of $X_{t}=\left(X_{t}^{(1)}, X_{t}^{(2)}\right)$ \\
$F_{a t}$ & c.d.f of a Gamma distributed r.v. with parameters $(a t, 1)$ \\
$\bar{F}_{a t}$ & s.f. of a Gamma distributed r.v. with parameters $(a t, 1)$ \\
\hline
\end{tabular}

Bérenguer, C., Grall, A., Dieulle, L., \& Roussignol, M. (2003). Maintenance policy for a continuously monitored deteriorating system. Probab. Engrg. Inform. Sci 17(2): 235-250.

Bertoin, J. (1996). Lévy processes. Cambridge Tracts in Mathematics 121, Cambridge University Press, Cambridge.

Buijs, F. A., Hall, J. W., Van Noortwijk, J. M., \& Sayers, P. B. (2005). Time dependent reliability analysis of flood defences using Gamma processes. In Augusti, G., Schüeller, G. I., \& Ciampoli, M., editors, Safety and Reliability of Engineering Systems and Structures; Proceedings of ICOSSAR 9: 22092216, Rotterdam. Millpress.

Cherian, K. C. (1941). A bi-variate correlated gamma-type distribution function. J. Indian Math. Soc. (N.S.) 5: 133-144.

Devroye, L. (1986). Non-uniform random variate generation. SpringerVerlag, New York. 
Ebrahimi, N. (2004). Indirect assessment of the bivariate survival function. Ann. Inst. Statist. Math. 56(3): 435-448.

Eymard, R. \& Mercier, S. (2008). Comparison of numerical methods for the assessment of production availability of a hybrid system. Reliab. Eng. Syst. Saf. 93(1): 168-177.

Mercier, S., Meier-Hirmer, C., \& Roussignol, M. (2012). Bivariate Gamma wear processes for track geometry modelling, with application to intervention scheduling. Struct. Infrastruct. E. 8(4): 357-366.

Sato, K.-I. (1999). Lévy processes and infinitely divisible distributions. Cambridge Studies in Advanced Mathematics 68, Cambridge University Press, Cambridge.

Singpurwalla, N. (1995). Survival in dynamic environments. Statist. Sci. 10(1): 86-103.

Van Noortwijk, J. (2009). A survey of the application of Gamma processes in maintenance. Reliab. Eng. Syst. Saf. 94(1): 2-21. 